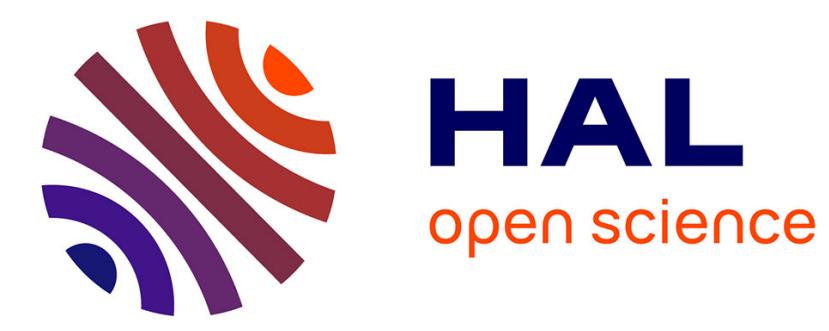

\title{
Plasma beam characterization along the magnetic nozzle of an ECR thruster
}

\author{
Sara Correyero, J. Jarrige, D. Packan, Eduardo Ahedo
}

\section{To cite this version:}

Sara Correyero, J. Jarrige, D. Packan, Eduardo Ahedo. Plasma beam characterization along the magnetic nozzle of an ECR thruster. Plasma Sources Science and Technology, 2019, 28 (9), pp.095004. 10.1088/1361-6595/ab38e1 . hal-02794843

\section{HAL Id: hal-02794843 \\ https://hal.science/hal-02794843}

Submitted on 5 Jun 2020

HAL is a multi-disciplinary open access archive for the deposit and dissemination of scientific research documents, whether they are published or not. The documents may come from teaching and research institutions in France or abroad, or from public or private research centers.
L'archive ouverte pluridisciplinaire HAL, est destinée au dépôt et à la diffusion de documents scientifiques de niveau recherche, publiés ou non, émanant des établissements d'enseignement et de recherche français ou étrangers, des laboratoires publics ou privés. 
ACCEPTED MANUSCRIPT

\section{Plasma beam characterization along the magnetic nozzle of an ECR thruster}

To cite this article before publication: Sara Correyero et al 2019 Plasma Sources Sci. Technol. in press https://doi.org/10.1088/1361-6595/ab38e1

\section{Manuscript version: Accepted Manuscript}

Accepted Manuscript is "the version of the article accepted for publication including all changes made as a result of the peer review process, and which may also include the addition to the article by IOP Publishing of a header, an article ID, a cover sheet and/or an 'Accepted

Manuscript' watermark, but excluding any other editing, typesetting or other changes made by IOP Publishing and/or its licensors"

This Accepted Manuscript is @ 2019 IOP Publishing Ltd.

During the embargo period (the 12 month period from the publication of the Version of Record of this article), the Accepted Manuscript is fully protected by copyright and cannot be reused or reposted elsewhere.

As the Version of Record of this article is going to be / has been published on a subscription basis, this Accepted Manuscript is available for reuse under a CC BY-NC-ND 3.0 licence after the 12 month embargo period.

After the embargo period, everyone is permitted to use copy and redistribute this article for non-commercial purposes only, provided that they adhere to all the terms of the licence https://creativecommons.org/licences/by-nc-nd/3.0

Although reasonable endeavours have been taken to obtain all necessary permissions from third parties to include their copyrighted content within this article, their full citation and copyright line may not be present in this Accepted Manuscript version. Before using any content from this article, please refer to the Version of Record on IOPscience once published for full citation and copyright details, as permissions will likely be required. All third party content is fully copyright protected, unless specifically stated otherwise in the figure caption in the Version of Record.

View the article online for updates and enhancements. 


\title{
Plasma beam characterization along the magnetic nozzle of an ECR thruster
}

\author{
S. Correyero ${ }^{1}$, J. Jarrige ${ }^{2}$, D.Packan ${ }^{2}$, E. Ahedo ${ }^{1}$ \\ ${ }^{1}$ Equipo de Propulsión Espacial y Plasmas, Universidad Carlos III de Madrid, Leganés, Spain, \\ ${ }^{2}$ ONERA, The French Aerospace Laboratory, Palaiseau, Paris, France, \\ Email:scorreye@uc3m.es.
}

\begin{abstract}
:
Experimental characterization of plasma properties along the magnetic nozzle of an Electron Cyclotron Resonance thruster is presented here. A permanent magnet prototype and a solenoid prototype are tested, whose main difference relies on the magnetic field strength and topology. A cylindrical Langmuir probe is used to measure plasma potential, plasma density and electron temperature. In the permanent magnet thruster set-up, a Laser Induced Fluorescence diagnostics is performed simultaneously with the Langmuir probe to measure the mean ion kinetic energy, and a Faraday gridded probe to characterize the angular plasma beam. An effective electron cooling rate has been identified, as well as the dependence of the total plasma potential drop with the mass flow rate. Results are compared with a supersonic collisionless fluid-kinetic 1D model where electron dynamics account for magnetic mirror effects and potential barriers, while ions are treated as a fluid cold species. The comparison allows to estimate the sonic transition of the plasma flow.
\end{abstract}

\section{INTRODUCTION}

In simple terms, a magnetic nozzle (MN) consists of a divergent magnetic field generated by a a set of solenoids or permanent magnets, whose main function is to confine and accelerate the plasma beam. Interest in MNs has increased significantly during the past years, [1-3], accelerating the development of the so-called MN plasma thrusters, such as the helicon thruster $[2,4,5]$, the Variable Specific Impulse Magnetoplasma Rocket (VASIMR) [6], the applied-field magnetoplasmadynamic thruster $[7,8]$ or the electron cyclotron resonance (ECR) thruster [9,10]. Among its numerous advantages over other acceleration systems, noteworthy are its contactless character, which reduces erosion and can extend the thruster lifetime, its versatility which allows to adapt the magnetic field topology in flight or its easy scalability. However, plasma dynamics in a MN involve complex physical mechanisms that are yet not fully understood, and are crucial to determine the thruster performance. Several authors have contributed significantly to the development of this device, providing insightful theoretical and experimental research involving MN expansions, such as plasma detachment [11-15], formation of ambipolar electric field [16-18] and magnetic thrust generation [19-21]. Ideally, modelling and testing must make progress together, since one without the other may fall short under scientific scrutiny.

Experimental characterization of the plasma beam properties along a MN can reveal not only the thruster performance, but also important physical aspects concerning plasma expansion dynamics that are still unclear. 
Takahashi et al. in [22] studied the thermodynamics of a magnetized electron gas expansion, showing the correlation between an external electric field and the polytropic index. Little and Choueiri in [23] characterized the axial variation of macroscopic quantities in a RF plasma beam and investigated the electron cooling phenomena, comparing its results with a fluid 1D model that included electron heat conduction and revealing the critical need for improved 1D models to describe the MN expansion. More recently, experimental studies performed by Kim et al. in [24,25] showed the coexistence of adiabatic expanding electrons and isothermal confined electrons along the $\mathrm{MN}$ of an ECR thruster. In this sense, the requirement for accurate experimental data and validation of advanced theoretical models for MNs is clear.

On the experimental side, ONERA has been developing an ECR thruster during the past years consisting of an ECR coaxial cavity followed by a divergent MN [10]. Multiple research has been done concerning this technology: Vialis et al. performed the first direct thrust measurements in [21], an analytical model of the ECR source was developed to identify the most important physical features in the ECR discharge [26], Lafleur et al. performed a theoretical and experimental investigation in [27] to determine the relationship between the maximum ion energy and the upstream electron temperature in this device, founding a value of 4-5. In order to contribute to the previous research, and characterize longitudinally the plasma expansion along the center line of the ECR thruster, this work presents the first complete measurements of plasma potential, electron density, electron temperature, and ion velocity along the expansion. Two ECR prototypes are tested, with two different MN topologies. A parametric study of variation of the mass flow rate $\dot{m}$ is presented, which is crucial to understand the expansion dynamics, since the electron temperature and the isotropization of the electron energy distribution function are highly dependent on this parameter. Langmuir probes have been used to characterize the evolution of plasma properties along the axis of the MN, and a Laser Induced Fluorescence (LIF) diagnostics has been employed to determine the mean ion/velocity in the thruster exit plane and along the center line of the expansion [28]. Angular scans of the ion current have been performed at different xenon mass flow rates to estimate thruster performances partially.

The fluid-kinetic quasi-1D steady-state model by Martinez-Sanchez, Ahedo and Navarro [29] is here adapted to analyze the experimental data along the MN of the thruster. The model determines self-consistently the axial profile of the ambipolar electric potential and the electron distribution function of a magnetized fully-divergent plasma expansion. Contrary to the commonly used fitted polytropic laws, the model in [29] determines directly the exact solution of the electron velocity distribution function and the total potential drop in the magnetized expansion, which for near-cold ions, only depends on the ion to electron mass ratio.

This paper is organized as follows: Section II explains the set-up developed at ONERA, Section III presents the main experimental results, Section IV describes the model used to predict the plasma properties along the expansion and compares the experimental data with the theoretical model. Finally, Section V concludes the main results of the research.

\section{EXPERIMENTAL SET-UP}

\section{II.1 The ECR thruster of ONERA}

The experiments carried out in this work were performed along the MN of the ECR thruster developed by ONERA [10]. It has an axisymmetric geometry and it is composed of a coaxial plasma source cavity of 
dimensions $L_{\mathrm{s}}=15 \mathrm{~mm}$ and $R_{\mathrm{s}}=13.5 \mathrm{~mm}$ followed by a divergent MN. $2.45 \mathrm{GHz}$ electromagnetic waves are emitted by a stainless steel antenna of $0.9 \mathrm{~mm}$ radius placed at the thruster axis and the electron resonance condition is achieved at $875 \mathrm{G}$. Two different prototypes are tested, were the magnetic field is either generated by a solenoid $(\mathrm{SO})$ or by a permanent magnet (PM) named ECR-PM-V1 in [21]. The back of the source is limited by a dielectric back plate made of boron nitride. The thrusters are electrically isolated by an inline microwave component called Direct Current Blocker (DC block), which allows them to be electrically floating. The propellant is injected at the back plate of the source through two holes of 2 mm diameter located symmetrically at two mid-points of the source radius. The external magnetic field strength at the thruster back plate is fixed at $900 \mathrm{G}$ for both prototypes, but the PM thruster has a smaller axial gradient, which shifts downwards the plasma detachment and results in a less divergent plasma beam. Figure 1 compares the magnetic field strength at the thruster's axis as a function of the distance to the thruster exit plane (corresponding to $z=0)$.

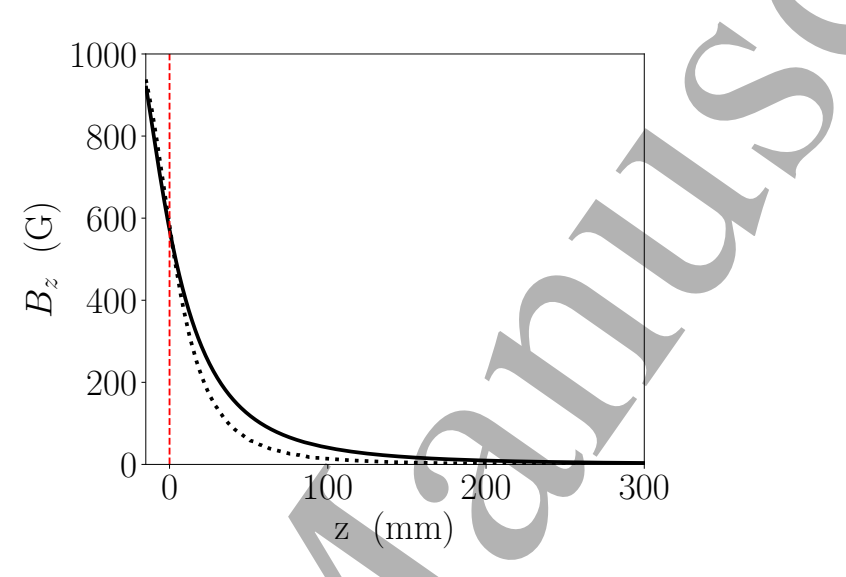

Figure 1: Applied magnetic field strength at thruster axis in the PM ( - ) and SO ( .....) ECR prototypes. $z=0$ corresponds to the thruster exit plane.

\section{II.2 Subsystems and operating conditions}

The microwave power is injected by means of a solid state amplifier. It is constituted by a signal generator (between 2.3 and $2.6 \mathrm{GHz}$ ) and a linear amplifier, and the maximum power it can deliver is around $100 \mathrm{~W}$. The power is transmitted by a 50 Ohm coaxial cable, whose losses have been measured using a vector network analyzer. The output of the generator is connected directly to a circulator with a 50 Ohm load, which allows the reflected power to be absorbed and dissipated in the load. Subsequently and before entering the feedthrough, the power line contains a bi-directional coupler connected to two different diodes that are in charge of measuring the forward and reflected power at this point of the line. Figure 2 shows a schematic of the the microwave power line set-up.

In this study, the power absorbed by the plasma was set at $30 \pm 2 \mathrm{~W}$ (determined by recording the input and reflected power measured by the bi-directional coupler). The measured reflected power was less than $3 \%$ in all the cases presented here. The level of radiated power was evaluated when the thruster was ignited and was found to be negligible and the attenuation level in the coaxial cable inside the chamber was characterized with a Vector Network Analyzer. With this procedure, the absorbed power was estimated to be very close to the 


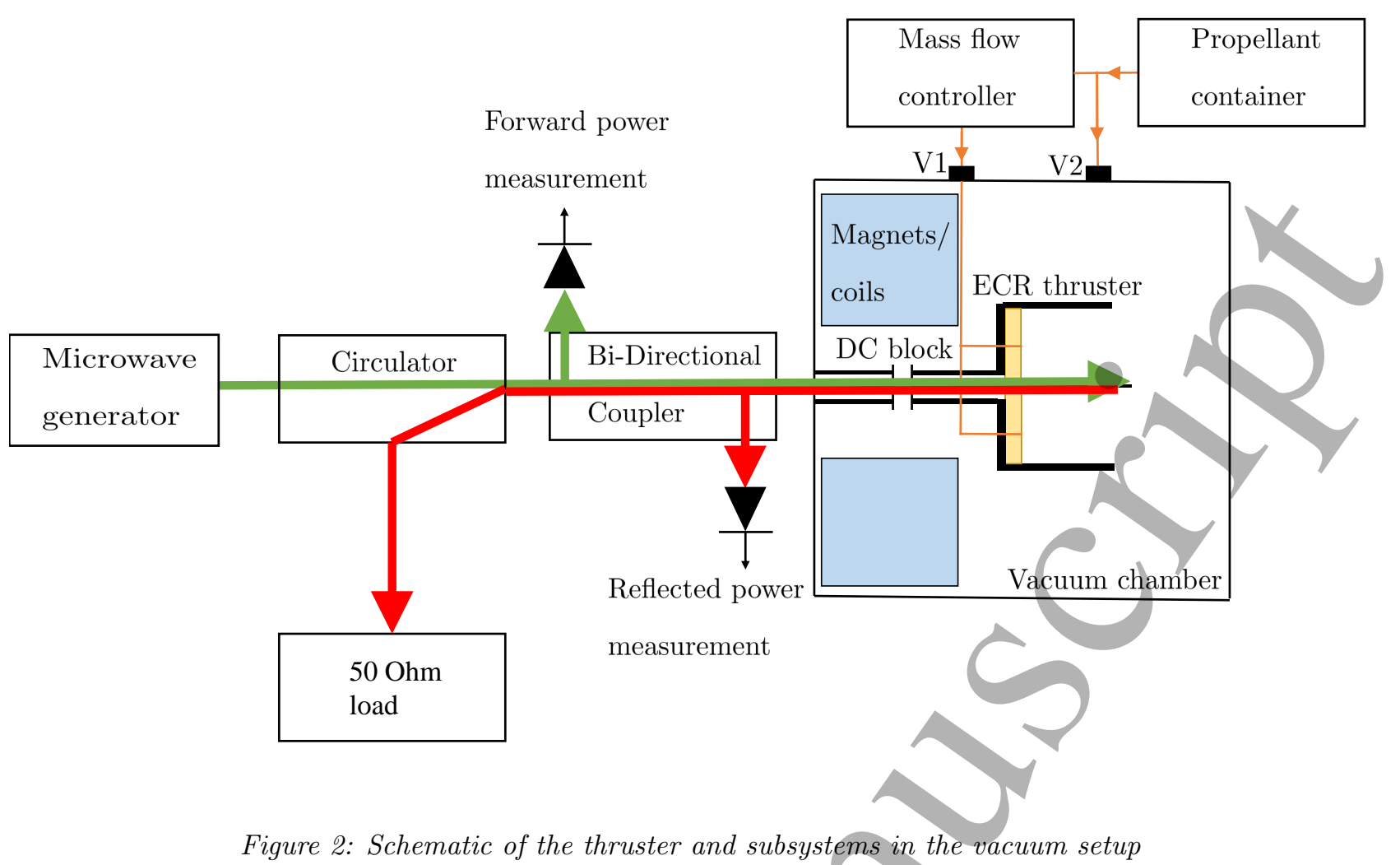

input power in this set-up $(\approx 95 \%)$. Finally, the xenon mass flow rate varied within the range $\dot{m}=(0.08-0.2)$ $\mathrm{mg} / \mathrm{s}$.

The tests have been done at ONERA-Palaiseau facilities. The vacuum chamber, known as B09, consists of a cylindrical vessel with dimensions of $0.8 \mathrm{~m}$ diameter and $2 \mathrm{~m}$ long. The pumping is done by one primary pump, three Pfeiffer Hipace $\left(2000 \mathrm{l} / \mathrm{s} \mathrm{N}_{2}\right.$ ) turbomolecular pumps, and one cryogenic pump, allowing to achieve a background pressure of $10^{-7}$ mbar and a xenon pumping speed of $13000 \mathrm{l} / \mathrm{s}$.

\section{II.3 Diagnostics}

In order to characterize longitudinal profiles of the plasma properties along the MN expansion, two different diagnostics were installed: Laser Induced Fluorescence (LIF) optical set-up to measure the ion velocity distribution function (IVDF) and cylindrical Langmuir probes to obtain information about the electron energy probability function (EEPF). Additionally, a Faraday gridded probe was installed in a rotation stage placed at $28 \mathrm{~cm}$ from the thruster exit plane to perform angular scans of the total ion current. The different diagnostics are detailed below.

\section{II.3.1 Cylindrical Langmuir probe}

A cylindrical Langmuir probe of $0.1 \mathrm{~mm}$ diameter and $6 \mathrm{~mm}$ length was placed in a 2D translation stage, so it could operate at different longitudinal positions. The tip probe was made of tungsten wire and it was held in a ceramic tube. The I-V characteristic curve was successfully obtained at different locations without perturbing significantly the thruster performance (from 75 to $250 \mathrm{~mm}$ from the thruster exit plane for the 
PM prototype, and from 40 to $280 \mathrm{~mm}$ for the SO prototype) and local values of plasma potential, electron density and electron temperature were computed. It was not possible to place the probe closer to the thruster, because significant variations on the floating thruster potential were detected, an indication that the diagnostic was interfering the thruster performance. The floating thruster potential was measured using a commercial multimeter external to the vacuum chamber.

One of the main concerns when placing a Langmuir probe in the presence of a magnetic field is the tip orientation with respect to the magnetic field lines. The Larmor or gyro radius was expected to be greater than the probe radius in all the range studied here, so no magnetic field effect should in principle be captured [30]. To ensure this fact, and since the gyro radius was not well known due to the uncertainty on the electron temperature, a previous test was performed with the Langmuir probe placed in a rotation stage, Acquisitions were taken with the probe placed parallel and perpendicular to the magnetic field lines and no significant difference in the I-V curve was found. For this reason, the effect of the probe orientation was neglected in the present work, and the electron temperature shown here should be understood as a total value of the electron energy (including parallel and perpendicular orientations).

\section{II.3.2 LIF}

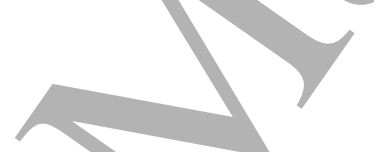

A 1D Laser Induced Fluorescence diagnostic was mounted together with the electrostatic probes (Langmuir and Faraday gridded) to measure the projection of the ion velocity distribution function along the thruster axis direction. All optics were fixed to the vacuum chamber, and the thruster was moved using a 3D translation stage. With this setup, any point in the thruster source and along the plume could be measured. It must be noticed that this diagnostic was only installed when operating the PM thruster, since the SO prototype includes water cooling tubes and high current cables $(130 \mathrm{~A})$ that were not compatible with the 3D translation stage.

The LIF calibration and specific details of the optical setup are explained in [28], as well as the main results from the LIF measurements presented in this work. The laser consists of a SDL-TC10 tunable laser diode with $20 \mathrm{~mW}$ of maximum output power and the frequency span for this measurements was $25 \mathrm{GHz}$, which allowed measuring axial ion velocities up to $20 \mathrm{~km} / \mathrm{s}$.

The laser was aligned with the thruster axis and injected through a lens of $25 \mathrm{~mm}$ diameter. The detection was composed of a $50 \mathrm{~mm}$ lens and a $200 \mu \mathrm{m}$ optic fiber. The probed volume was around $1 \mathrm{~mm}$ diameter. The optics were installed $450 \mathrm{~mm}$ from the measurement point, to avoid perturbation and intrusion in the plasma beam. The detection was installed with an angle of $35^{\circ}$ and a camera was fixed to the thruster to verify alignment between the laser and the detection. Figure 3 schematizes the set-up. 


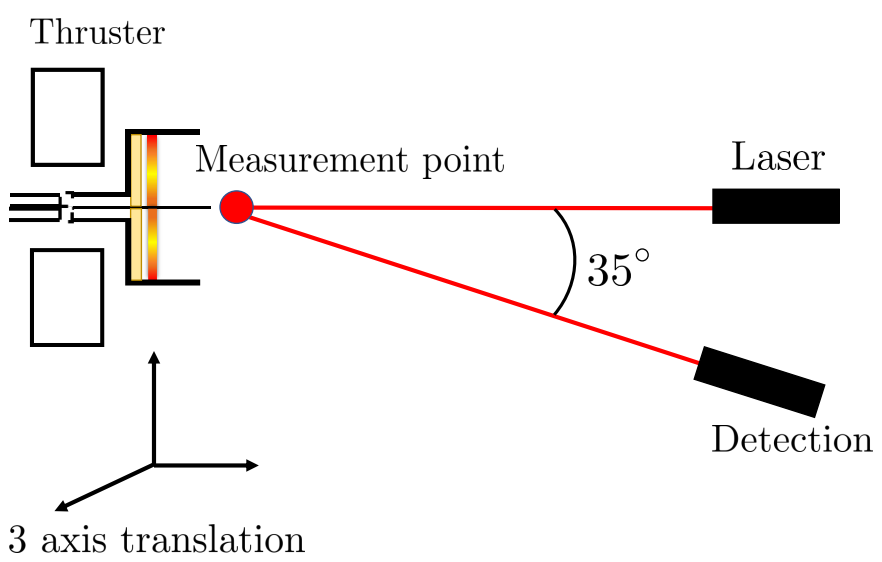

Figure 3: Schematic of optical LIF setup inside the vacuum chamber

\section{II.3.3 Faraday gridded probe}

To estimate the mass utilization efficiency at different mass flow rates, the total ion current was measured with a one-grid Faraday probe. Angular profiles of the ion current density/were obtained by performing angular scans of current with the probe mounted in a rotation/stage at $28 \mathrm{~cm}$ from the thruster exit plane. The beam is assumed to be axisymmetric and composed by only single ionized ions. The probe used here is described in [27], and consists of a $6 \mathrm{~mm}$ diameter collector with a front grid of $60 \%$ transparency. The voltage was biased at a sufficiently negative value to ensure the probe was working in the ion saturation region of the I-V curve. The procedure was the following: The probe was placed facing the plume at the thruster axis, and the collector was biased more negatively until there was no variation in the collected current $(\approx-300 \mathrm{~V})$. Then, the ion current was estimated by means of an external resistor. The Faraday probe was only mounted in the PM prototype setup.

\section{RESULTS}

This Section presents the results of the two magnetic systems setups used for the ECR thruster. All the tests presented here were carried out at $30 \pm 2 \mathrm{~W}$ of absorbed power and xenon mass flow rates of $1,1.5,2.0$ sccm for the PM thruster and 0.8,1 sccm for the SO thruster. In both setups, the Langmuir probe described in Section II was installed in a translation stage which allowed the probe tip to be aligned with the thruster axis at different axial positions. Additionally, in the PM thruster setup, the LIF optical assembly and the Faraday probe described in Section II were also installed. In this Section results are organized by diagnostic.

\section{III.1 Langmuir probe: Plasma properties $\phi, n_{e}, T_{e}$}

The plasma potential at each axial position was obtained from the inflection point of the I-V characteristic curve, and the electron energy distribution function (EEDF) was computed with the Druveysteyn formula [31], 
shown in equation 1, where $F(\epsilon)$ represents the EEDF, $g(\epsilon)$ the electron energy probability function (EEPF), $m_{e}$ and $q$ the electron mass and charge respectively and $A$ the probe surface area. Figure 4 shows an example of the I-V curve and the calculated EEPF at $1 \mathrm{sccm}$, at $105 \mathrm{~mm}$ from the PM thruster exit plane. The electron density and temperature can be computed by taking the corresponding integrals of the EEDF, as it is shown in equations 2 and 3. Notice that since the EEPF is not Maxwellian, $T_{e}$ stands for an effective electron temperature. These formulas give reliable results when a magnetic field is present if the condition $r_{L}>>r_{s}$ is satisfied, where $r_{L}$ represents the electron Larmor radius or gyroradius and $r_{s}$ the effective collecting area of the probe. This assumption can be problematic at the closest locations to the thruster exit plane, since the electron Larmor radius could remain of the same order as the probe sheath length. For instance, for the SO thruster, at $\mathrm{z}=80 \mathrm{~mm}$ the $r_{L}$ expected should be around $4 \mathrm{~mm}$, while the estimated $r_{s}$ is $2.61 \mathrm{~mm}$. However, in most of the analyzed region, the condition $r_{L}>>r_{s}$ is well satisfied.
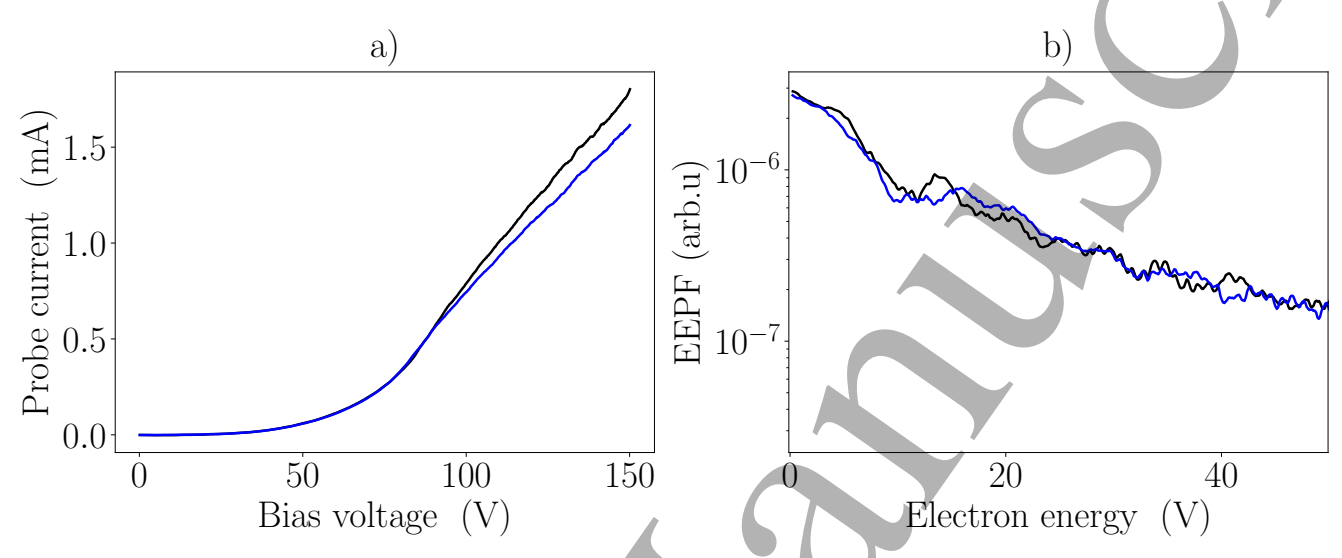

Figure 4: a) I-V curves at 2 sccm of xenon, at different axial locations from the PM thruster exit plane and b) the computed EEPF.

$$
\begin{gathered}
F(\epsilon)=g(\epsilon) \sqrt{\epsilon}=\frac{2 \sqrt{2 m_{e} \epsilon}}{q^{3} A} \frac{d^{2} I}{d V^{2}} \\
n_{e}=\frac{2 \sqrt{2 m_{e}}}{|q| A} \int_{0}^{-\infty} \frac{d^{2} I}{d V^{2}} \sqrt{\frac{V}{|q|}} d V \\
T_{e}=\frac{4 \sqrt{2 m_{e}}}{3 n_{e} A} \int_{0}^{-\infty} \frac{d^{2} I}{d V^{2}}\left(\frac{V}{|q|}\right)^{3 / 2} d V
\end{gathered}
$$

Figure 5 shows the plasma properties measured with the Langmuir probe along the center line of the PM thruster plume. Plasma potential $\phi$, electron density $n_{e}$ and electron temperature $T_{e}$ have been estimated from 75 mm to $250 \mathrm{~mm}$ downstream, at three different xenon mass flow rates $1,1.5$ and $2 \mathrm{sccm}$, where $z=0$ corresponds to the thruster exit plane. The plasma potential $\phi$ has been plotted referred to its value at $z=75 \mathrm{~mm}$. Figure 5 (a) shows there is a direct relation between the mass flow rate and the plasma potential profile in the studied region: at higher mass flow rates, lower is the plasma potential drop. This behaviour is directly related to the electron temperature at the plasma source [27], which is highly dependant on the mass flow rate.

The spatial range of the Langmuir probe measurements was limited to $250 \mathrm{~mm}$ from the thruster exit plane due to the translation stage length. Moreover, positioning the probe closer than $75 \mathrm{~mm}$ from the exit 
plane disturbed significantly the thruster performance. For this reason, within 0-75 mm, the experiment was considered highly intrusive. Although the plasma potential profiles have been found to be almost flat beyond $200 \mathrm{~mm}$, upstream of $75 \mathrm{~mm}$ a high ambipolar potential gradient is expected, and therefore the near region can not be neglected to estimate the entire plasma potential profile curve. In the ECR thruster tested here, with its particular magnetic field topology, the first $10 \mathrm{~cm}$ down from the thruster exit plane are crucial to the ions acceleration (and therefore to the plasma potential), as it is confirmed in Section III.2. In order to complete the near region of the plasma potential profile, the LIF optical setup was installed, total ion energy was assumed to be conserved, and the plasma potential in the near region was estimated by means of the LIF diagnostics.

Figure 5 (b) shows the estimated electron density $n_{e}$ measured with the Langmuir probe. As it is expected, it shows an increase with the mass flow rate. At $75 \mathrm{~mm}$, densities in the order of $10^{15} \mathrm{~m}^{-3}$ have been found. Finally, the electron temperature $T_{e}$ shows the opposite behaviour; it decreases with the mass flow rate (see figure 5 (c)). The total absorbed power measured for all cases is $30 \mathrm{~W}$, so for lower mass flow rates, more energy is spent in heating the electrons, instead of ionizing the plasma. An effeetive electron cooling has been measured along the expansion at different operating conditions. To identify the electron cooling rate, the electron temperature has been plotted against the electron density in a logarithm scale in figure 5 (d). Polytropic coefficients of $1.23 \pm 0.02$, have been found, all of them below the adiabatic expansion coefficient $5 / 3$ and similar to the values found by other authors in magnetically expanding plasmas [23,32]. However, this value only corresponds to a delimited region of the expansion.
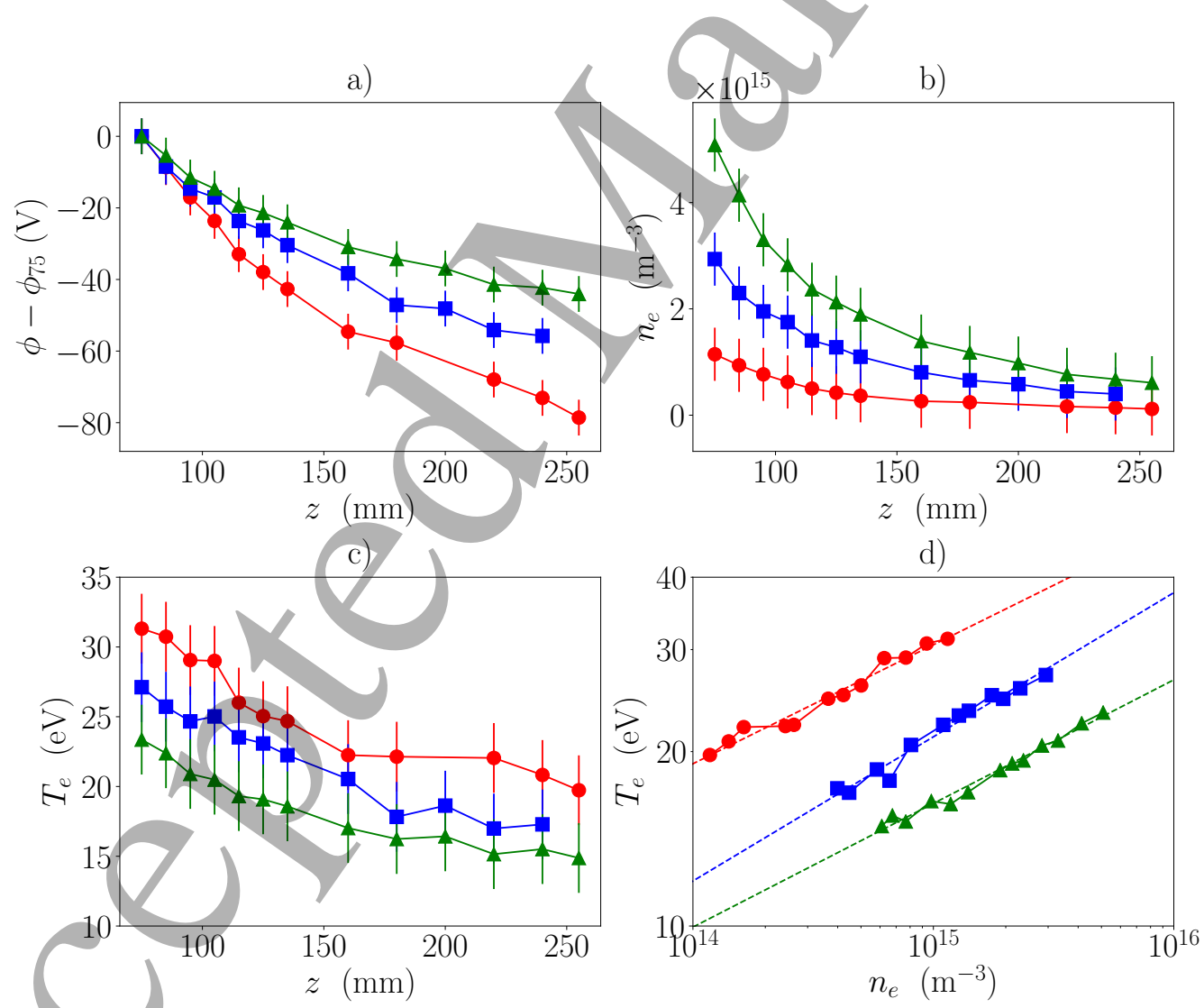

Figure 5: Plasma properties measured with the Langmuir probe, along the center line of the PM thruster. $z=0$ corresponds to the thruster exit plane. Measurements were taken at $30 \mathrm{~W}$ of power and different mass flow rates: $1 \mathrm{sccm}(-) 1.5 \mathrm{sccm}(-), 2 \mathrm{sccm}(-)$ ). 
Experimentally, covering the whole region of the expansion can add insight on the electron cooling mechanism, and in this sense, the Langmuir probe measurements in the SO thruster plume represent a farther zone of the MN, where there is a higher drop of the magnetic field strength. Figure 6 shows the plasma properties measured with the Langmuir probe along the magnetic field expansion of the SO thruster. The Langmuir probe was operated from $z=40$ to $z=290 \mathrm{~mm}$. In this experiment, the thruster performance was found stable from $40 \mathrm{~mm}$ downwards. As it can be observed in figure 6 (a), in the range from $z=75 \mathrm{~mm}$ to $z=250 \mathrm{~mm}$, at 1 sccm, the plasma potential has decreased around $35 \mathrm{~V}$, while in the same spatial region for the PM thruster, the total drop was around $80 \mathrm{~V}$. This is due to the fact that the acceleration region for the PM thruster is wider than for the SO thruster, which is a direct consequence of the MN topology. Concerning the plasma density/; a factor of two has been found between the SO thruster and the PM thruster for the same operation conditions, being higher in the SO thruster plume (see figure $6(\mathrm{~b})$ ).

Interestingly, the electron temperature profile measured in the SO thruster plume differs from the tendency measured along the nozzle of the PM thruster. As it is shown in figure 6 (c), it is possible to identify two different regions; while the first measurements from $z=40$ to $z=100 \mathrm{~mm}$ reveal a sharp decrease in the electron temperature, beyond $z=100 \mathrm{~mm}$ the curve remains almost flat, reaching an asymptotic value around $10 \mathrm{eV}$. In order to study this behaviour, the electron temperature is plotted against the electron density in a logarithm scale (figure 6 (d)). In contrast to the PM thruster, two different polytropic coefficients $\gamma$ have been identified here, $\gamma=1.55$ close to the thruster exit and $\gamma=1.17$ farther downstream. Similar trends have been found at $0.8 \mathrm{sccm}(-\bullet)$ and $1 \mathrm{sccm}\left(\bullet^{-}\right)$of xenon in the SO thruster plume, being the total plasma potential drop at $0.8 \mathrm{sccm}$ slightly higher. The experiments by Kim et. al in $[24,25]$ also found various values of the polytropic index along a MN expansion. In conclusion, these results indicate that the plasma expansion is more complex than polytropic. Indeed, the the MN model briefly presented later will confirm that there is not a simple polytropic law.

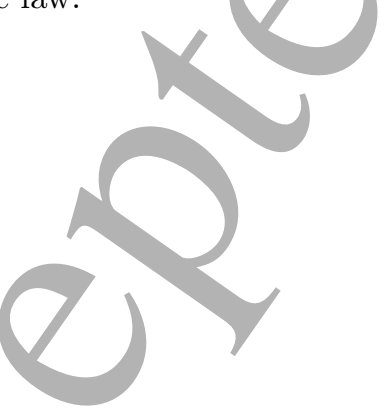

In figure 7 the electron density has been plotted against the plasma potential in logarithm scale, and compared to the Boltzmann relation and the different polytropic cooling laws. In the PM thruster (figure 7 (a)), a polytropic cooling law represents well the near region of the expansion, but downstream it leads to a non-negligible deviation. In the SO thruster plume (figure 7 (b)), the non local behaviour (i.e. change in polytropic factor) is again visible. 
a)

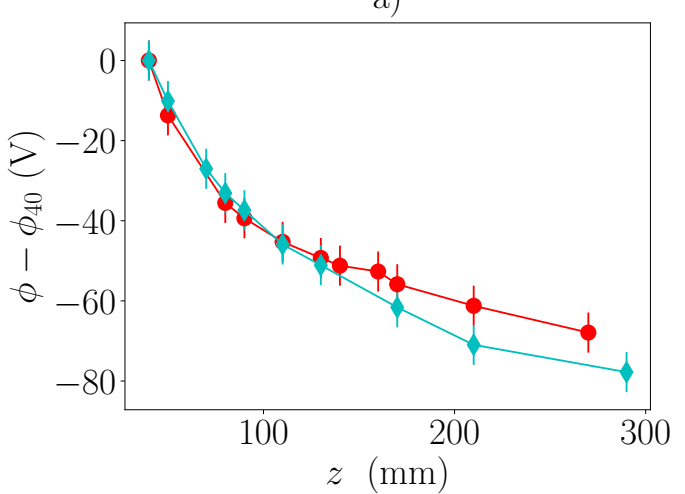

c)

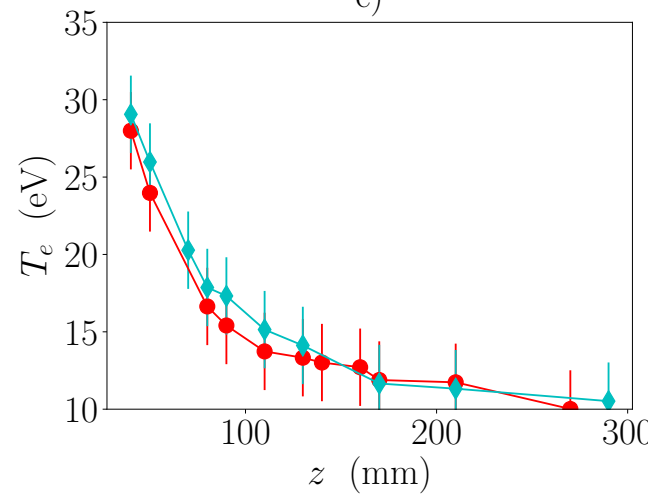

b)

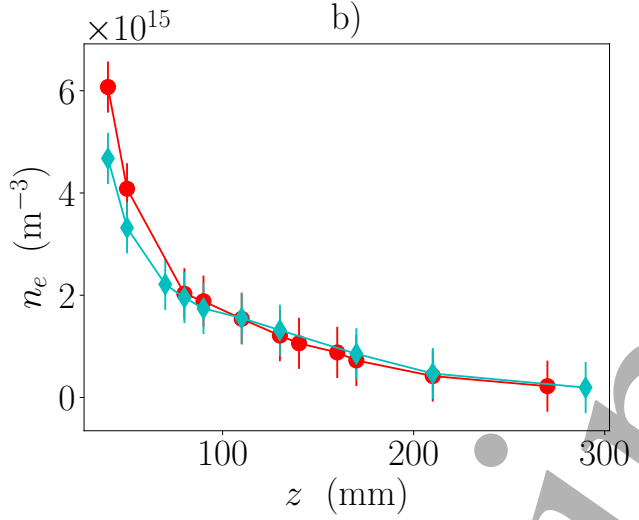

d)

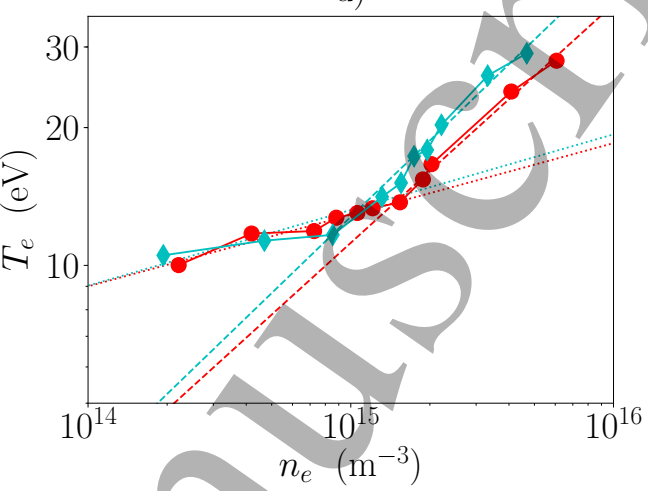

Figure 6: Plasma properties measured with the Langmuir probe, along the center line of the SO thruster. $z=0$ corresponds to the thruster exit plane. Measurements were taken at $30 \mathrm{~W}$ of power and different mass flow rates: $0.8 \operatorname{sccm}(\multimap) 1 \operatorname{sccm}(\multimap)$

a)

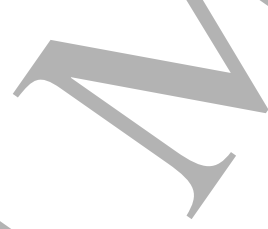

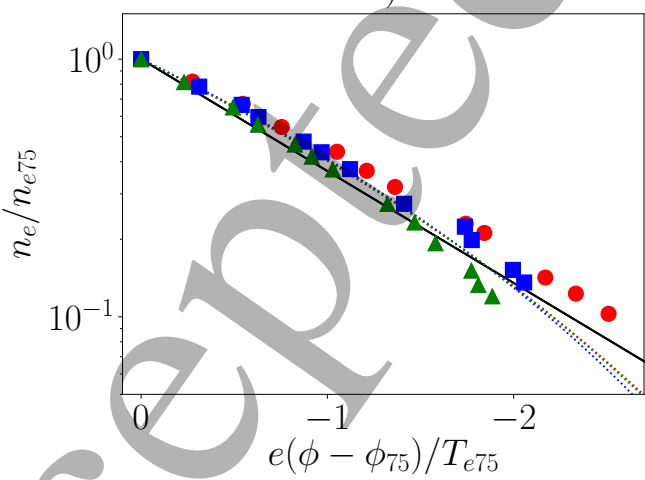

b)

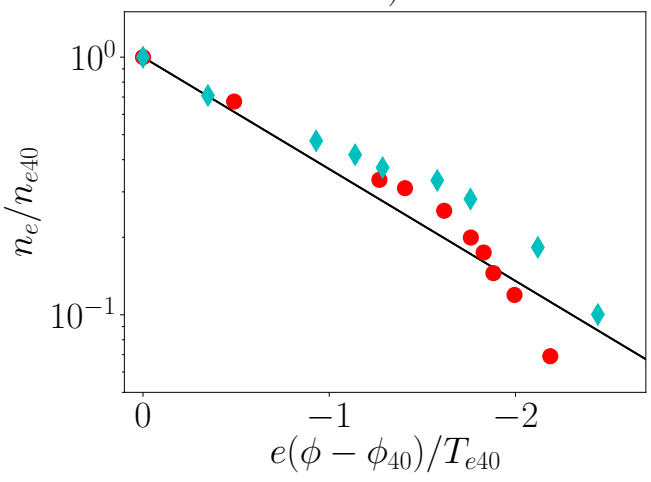

Figure 7: Normalized plasma density $n_{e} / n_{e 75}$ versus $e\left(\phi-\phi_{75}\right) / T_{e 75}$ estimated with the Langmuir probe for a) PM thruster and Normalized plasma density $n_{e} / n_{e 40}$ versus $e\left(\phi-\phi_{40}\right) / T_{e 40}$ b) SO thruster. 0.8, 1, 1.5, and 2 sccm are represented by $(\diamond),(\bullet),(\bullet),(\bullet)$, respectively. The solid line corresponds to the Boltzmann expansion, while the dashed lines in a) represent polytropic expansions with $\gamma=1.23 \pm 0.02$. 


\section{III.2 LIF: Mean axial ion velocity $u_{i}$}

The optical LIF diagnostics were installed together with the Langmuir probe in the PM thruster set-up. The thruster was operated at $30 \mathrm{~W}$ and at three different mass flow rates. LIF measurements were performed at several axial positions along the center line of the thruster axis; inside the thruster source and in the near plume region (from $z=-5 \mathrm{~mm}$ and $z=125 \mathrm{~mm}$ ). Three examples of different LIF spectra obtained with the same acquisition parameters (sweep time of the laser and time constant of the lock-in amplifier) are shown in figure 8 (a). It is noteworthy that there is a broadening of the peaks inside the thruster, which could be either due to Zeeman splitting of the $834.7 \mathrm{~nm}$ transition, or to the production of charge ex-change ions. The possible effect of Zeeman splitting in this region is a current line of research. Relative density and mean axial velocity have been computed and represented in figure 8 (b). Since the ECR region is located near the backplate, and the magnetic field is fully divergent, the generated ions are already slightly accelerated inside the source. The measured ion velocity at the thruster exit plane is 2250,1950 and $1900 \mathrm{~m} / \mathrm{s}$ at 1, 1.5 and $2 \mathrm{sccm}$, respectively. Currently, an uncertainty of the ECR thruster performance is the location of the sonic transition from a subsonic plasma jet to a supersonic beam, due precisely to the coupling of ionization and acceleration processes, so obtaining accurate data of the ion velocity and density in this region can be crucial to understand this phenomenon. Figure 8 (b) shows a unexpected trend of the relative ion density between 5 and $10 \mathrm{~mm}$ from the thruster exit. These results could be due to production of secondary ions in this region, but it must be confirmed with additional measurements.

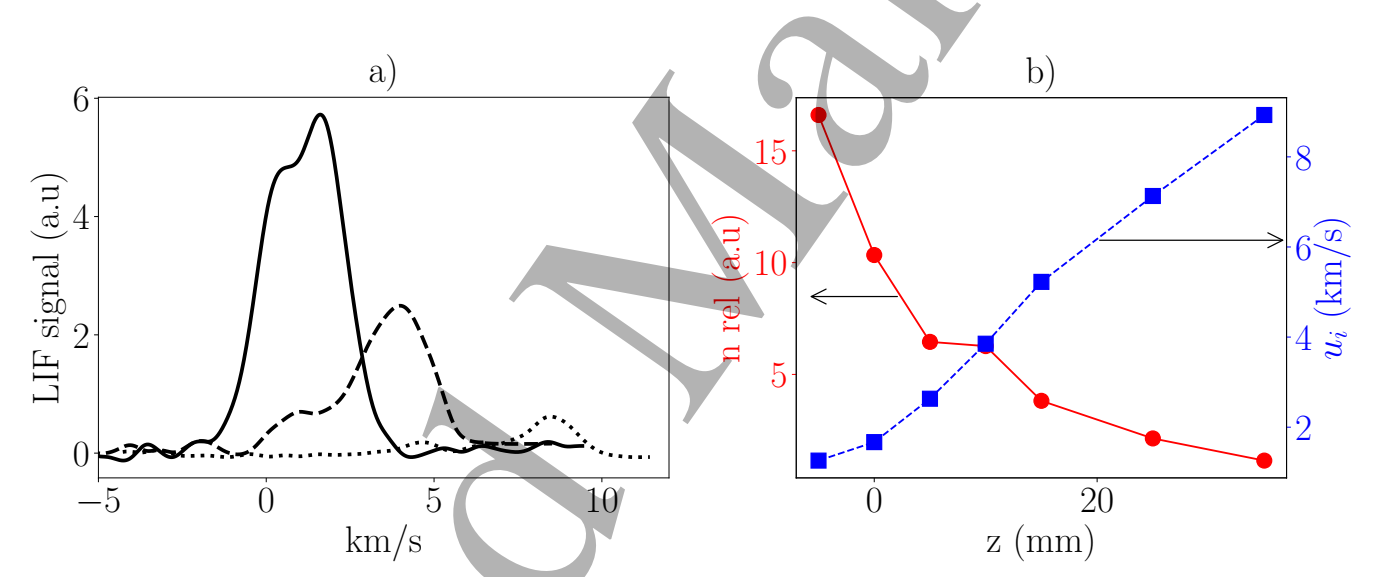

Figure 8: a) LIF signal already centered with the reference discharge at different axial positions: $-5 \mathrm{~mm}(-)$ ), $10 \mathrm{~mm}(---)$ and $35 \mathrm{~mm}(\cdots . .$.$) . b) Computed relative density ( -$ ) and mean axial velocity ( $\bullet-)$ from the LIF signals. Operating conditions were $1.5 \mathrm{sccm}$ and $30 \mathrm{~W}$.

The mean axial ion velocity has been determined from the LIF signal for each axial position, at $30 \mathrm{~W}$ of absorbed power and three different mass flow rates 1, 1.5 and $2 \mathrm{sccm}$. The longitudinal profiles are shown in figure 9 (a). Lower mass flow rates lead to higher ion velocities. The highest ion velocity measured at $100 \mathrm{~mm}$ from the thruster exit in this set-up was $16 \mathrm{~km} / \mathrm{s}$ for $1 \mathrm{sccm}$. However, the current density also decreases with the distance, and LIF measurements could not be performed beyond $100 \mathrm{~mm}$ at $1 \mathrm{sccm}$. The ion velocity axial gradient has been also plotted in figure 9 (b). It can be seen that the strongest gradient is located between 5 and $15 \mathrm{~mm}$ from the thruster exit plane, region where the electric field is more intense. As well, the maximum 
mass flow rates.

It can be seen that the acceleration region is not complete at the end of the LIF measurement range, henceforth LIF results in the near plume region have been completed with Langmuir probe measurements in the far plume region.
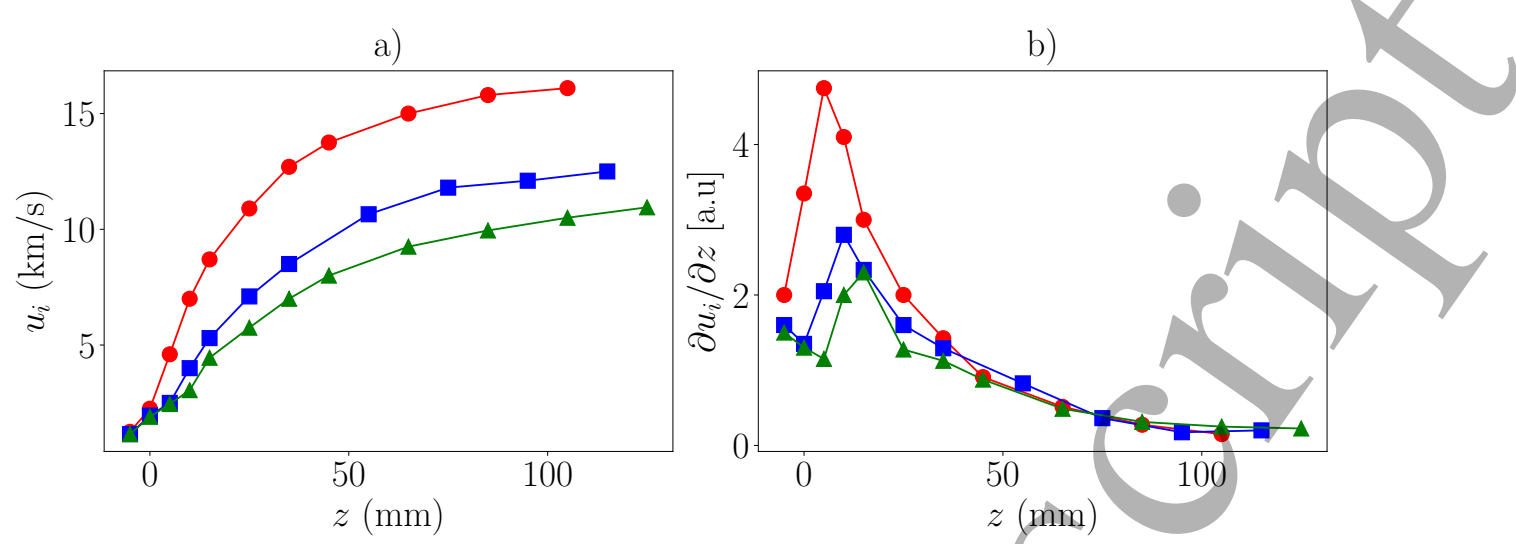

Figure 9: a) Mean axial ion velocity measured with the LIF set-up, along the center line of the PM thruster and b) its axial derivative normalized between 0 and 1. $z=0$ corresponds to the thruster exit plane. Measurements were taken at $30 \mathrm{~W}$ of power and different mass flow rates: $1 \mathrm{sccm}(-) 1.5 \mathrm{sccm}(\rightarrow-)$, $2 \mathrm{sccm}(-)$ ).

Finally, by assuming total ion energy is conserved, it is possible to calculate the plasma potential in the near plume from LIF measurements by overlapping the curves of kinetic energy and plasma potential in the region where LIF and Langmuir probe operated simultaneously. The resulting plasma potential profile from the thruster exit to the farthest measurement point, referred to the plasma potential at the ECR source is shown later in figure 14 (b). The total plasma potential drop from 0 to $250 \mathrm{~mm}$ is 236,145 and $104 \mathrm{~V}$, for 1 , 1.5 and $2 \mathrm{sccm}$, respectively. It is noteworthy that this potential drop is not necessarily the total ion energy, since although the plasma potential profile is almost flat beyond $200 \mathrm{~mm}$, the plasma expansion is not complete. One of the main contributions of this work is to compute the finite potential drop based on a fluid-kinetic plasma expansion model, and relate the percentage of plasma expansion with the spatial coordinates.

\section{III.3 Faraday probe: Ion current}

The gridded Faraday probe detailed in Section II.3.3 was installed in the PM thruster set-up. Angular scans of current density were taken at $28 \mathrm{~cm}$ from the thruster exit plane, at $30 \mathrm{~W}$ of absorbed power and three different mass flow rates 1, 1.5 and 2 xenon sccm. Figure 10 shows the measured angular profiles of ion current density, computed taking into account the surface of the probe and the grid transparency. It is noteworthy that the angular profiles of figure 10 do not reach the maximum current at 0 degrees, which is probably due to the fact that the antenna is located at the center line of the thruster, and influences the shape of the plasma beam. As well, the current design of the gas injection could also lead to non-homogeneities already in the plasma source, as it was pointed out by Vialis et al. in [21]. There is also a residual current at 90 degrees, which increases with the mass flow rate, which suggests that it could be due to charge ex-change collisions or additional ionization due to collisions between the ambient plasma and plume electrons.

The tơtal ion current $I_{i}$ is computed integrating the ion current density assuming an axisymmetric plume 


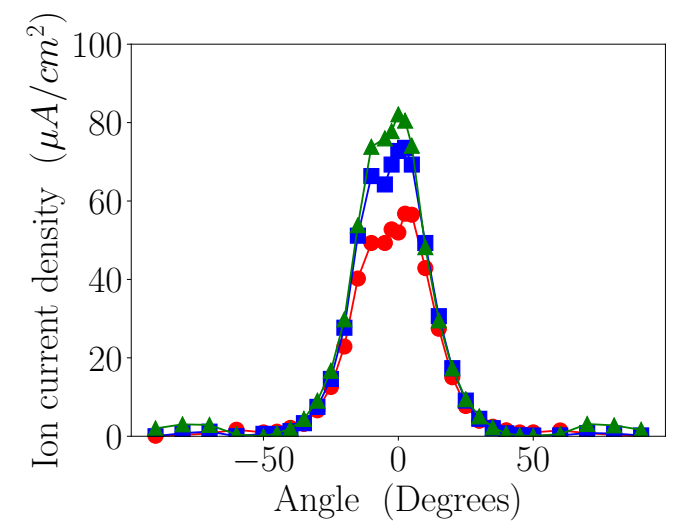

Figure 10: Angular scan of ion current density at $28 \mathrm{~cm}$ from the thruster exit: 1 sccm (- $-1.5 \mathrm{sccm}(\rightarrow-)$ and $2 \operatorname{sccm}(\longrightarrow)$.

and taking into account the distance to the thruster axis. Related to that current, a first parameter assessing the performance efficiency of the thruster is the mass efficiency, defined as

$$
\eta_{m}=\frac{I_{i} m_{i}}{\dot{m} q}
$$

where: $m_{i}$ is the ion mass, $q$ is the elementary electric charge, and $\dot{m}$ is the neutral gas mass flow rate. A second efficiency parameter is related to the plume divergence: if $\lambda$ represents an effective far field divergence angle [33], a "plume efficiency" is given by $\cos ^{2} \lambda$. Table 1 details these two performance parameters, together with the measured chamber pressure. The low measured mass efficiency is an indication of a poor ionization, which is directly related to the mass-flow rate to power ratio, as it was reported in [21].

\begin{tabular}{cccccc}
$\dot{m}(\mathbf{s c c m})$ & $p_{c h}\left(10^{-6} \mathbf{m b a r}\right)$ & $\eta_{m}(\%)$ & $\cos ^{2} \lambda$ & $T_{e *}(\mathbf{e V})$ & $z_{0}(\mathbf{m m})$ \\
\hline \hline 1 & 2.8 & 23.2 & 0.857 & 44 & 7.4 \\
1.5 & 3.5 & 17.6 & 0.892 & 27 & 11.8 \\
2 & 3.7 & 14.2 & 0.887 & 19 & 12.5
\end{tabular}

Table 1: Chamber pressure, mass utilization efficiency $\eta_{m}$ and plume efficiency $\cos ^{2} \lambda$ for the PM thruster. Two last columns: estimated electron temperature at the sonic point and its location for the MN model fitting.

\section{COMPARISON OF PLUME DATA WITH THEORY}

In order to understand and analyze the experimental data on the magnetized plasma plume emitted by the thruster, we resort to models of the MN. The most suitable one for a near-collisionless plasma is the kinetic one by Martínez-Sánchez et al. [29] which considers the expansion of a collisionless, quasineutral, magnetized plasma in a paraxial convergent-divergent MN, and solves for the ion and electron velocity distribution functions and the ambipolar electric potential. In the next Subsection, that ideal model is adapted to a divergent-only nozzle, in a way similar to the one Merino et al. [34] followed for an unmagnetized plasma plume. Then, Subsection IV.2 compares the experimental data with that model. 


\section{IV.1 Divergent nozzle model}

Let us consider a divergent nozzle created by an applied magnetic field $B(z)$ monotonically decreasing from $z=z_{0}$, where from a plasma beam is with near-sonic conditions, to be defined below. This plasma is collisionless, quasineutral, current-free and fully-magnetized. Therefore, ions and electrons are perfectly channeled by the magnetic streamtubes. The model, being paraxial, determines only axial flows of radiallyaveraged magnitudes. If the initial cross-section of the beam is $A_{0}$, the evolution of the cross section is inversely proportional to $B: A=A_{0} B_{0} / B$.

Individual electrons conserved their total energy $E_{e}$, and magnetic moment $\mu_{e}$, defined as

$$
\begin{gathered}
E_{e}=\frac{m_{e} w_{e \|}^{2}}{2}+\frac{m_{e} w_{e \perp}^{2}}{2}-e \Delta \phi \\
\mu_{e}=\frac{m_{e} w_{e \perp}^{2}}{2 B}
\end{gathered}
$$

where: $w_{e \|}$ and $w_{e \perp}$ are velocity components parallel (i.e. axial) and perpendicular to the magnetic field $\left(w_{e} \|\right.$ is also the electron gyrocenter velocity) and $\Delta \phi=\phi-\phi_{0} \leq 0$.

For a current-free plasma, the self-consistent electric potential $\phi$ is monotonically decreasing, its role being to adjust the downstream current of very mobile electrons to the ion current, in a similar way a Debye sheath does close to a dielectric wall. However, when electrons are magnetized there is an additional effect. Combining the above two equations, the electron axial velocity satisfies

$$
\frac{m_{e} w_{e \|}^{2}}{2}=E_{e}-\mu_{e} B+e \Delta \phi
$$

Therefore, the axial forward-motion $\left(w_{e \|}>0\right)$ of electrons is governed by two opposing forces: the electric one, which decelerates them, and the magnetic mirror effect, which accelerates them. The opposite is naturally true for back-marching electrons. As a consequence three different populations of electrons can be found in a divergent nozzle [35]: free electrons, emitted by the source and lost downstream (which constitute the electron current); reflected electrons, emitted by the source and turned back to the source; and doubly-trapped electrons, bouncing between two intermediate positions of the plume.

At the source, only the forward-marching velocity distribution function $f_{e+}\left(z_{0}\right)$ can be postulated, while the backward-marching distribution $f_{e-}(z)$ (at any location) is part of the solution. The semi-Maxwellian distribution

$$
\left.f_{e+}\left(E_{e}\right)\right|_{z_{0}}=n_{e *}\left(\frac{m_{e}}{2 \pi T_{e *}}\right)^{3 / 2} \exp \left(-\frac{E_{e}}{T_{e *}}\right), \quad w_{e \|}>0
$$

is chosen here. Parameters $n_{e *}$ and $T_{e *}$ would be the electron density and temperature at the source, $n_{e 0}$ and $T_{e 0}$, only if $f_{e+}=f_{e-}$. Since most source electrons are reflected back, $n_{e *} \approx n_{e 0}$ and $T_{e *} \approx T_{e 0}$. While the populations of free and reflected electrons are totally determined in terms of $B(z)$ and $\phi(z)$, the determination of the population of doubly-trapped electrons is out of the capabilities of a collisionless, stationary model. The same postulates than in [29] will be used here for that population. Macroscopic electron magnitudes such as density $n_{e}$, parallel particle flow $n_{e} u_{e}$, pressure $n_{e} T_{e}$, and parallel heat flux $q_{e}$, are determined as velocity moments of the local distribution function $f_{e}=f_{e+}+f_{e-}$, in the way explained in [29].

Ions also conserve their total energy and magnetic moment, but in this case, both the electric force and the magnetic mirror accelerate them downstream the divergent nozzle. As a consequence all ions constitute a 
free population, expanding supersonically, and a simple "cold fluid model" seems a good approximation [35]. Then, ion continuity and momentum equations are

$$
n_{i} u_{i} / B=\mathrm{const}=n_{i 0} u_{i 0} / B_{0}, \quad u_{i}=\sqrt{u_{i 0}^{2}-2 e \Delta \phi / m_{i}} .
$$

Using $B$ as independent variable instead of $z$, the self-consistent electric potential profile, $\phi(B)$, is determined by satisfying [29] plasma quasineutrality

$$
n_{e}(\phi(B), B)=n_{i}(\phi(B), B) \equiv n(B)
$$
and the current free condition, i.e. the (constant) ion and electron flows are identical. This, together with quasineutality states that macroscopic ion electron velocities are the same: $u_{e}(B)=u_{i}(B)$.

a)

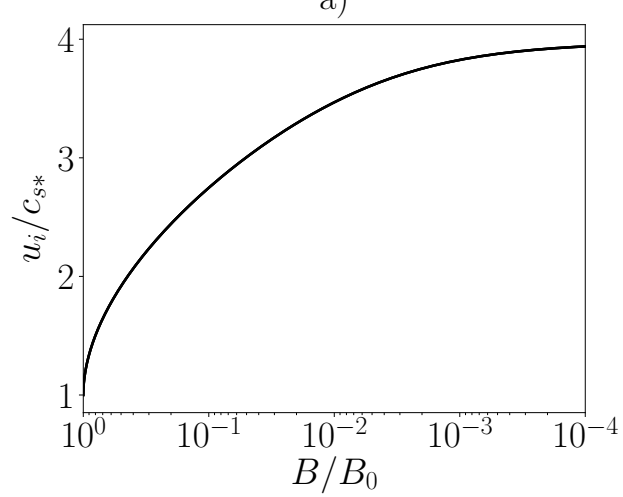

c)

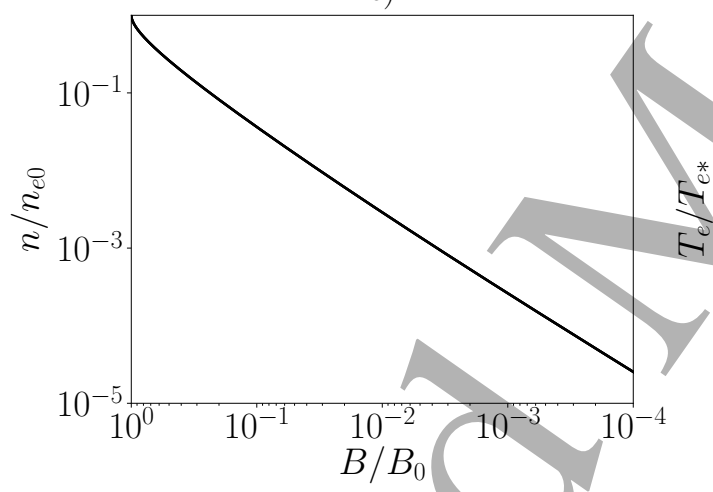

b)

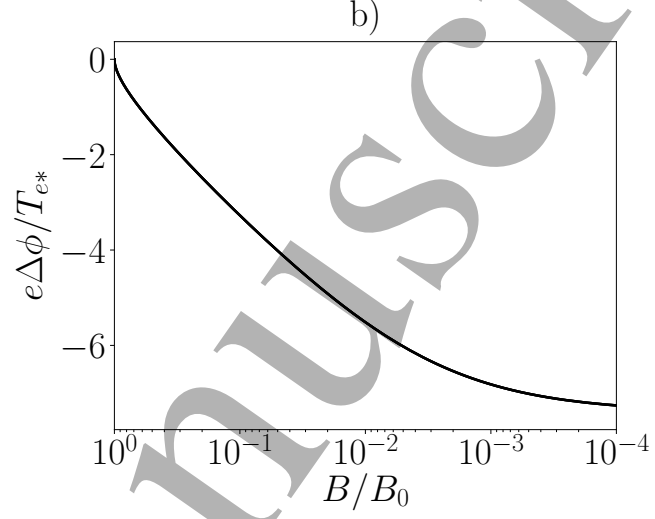

d)

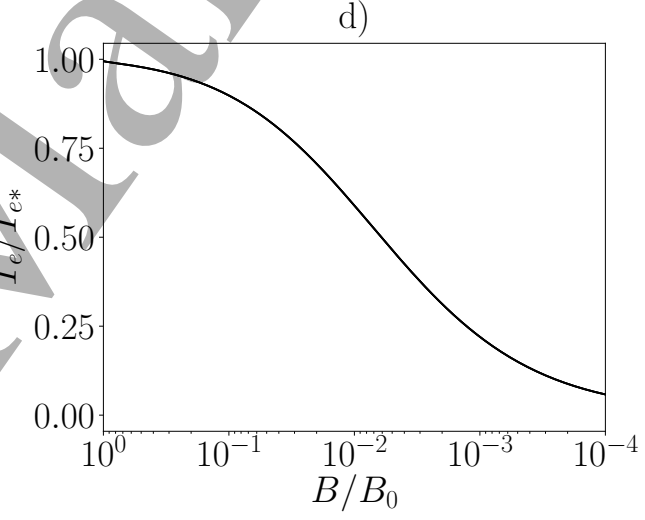

Figure 11: Plasma expansion according to the magnetic nozzle model.

The dimensionless solution of this problem depends on the injection location $z_{0}$ (or $B_{0}$ ), the initial velocity $u_{i 0} / c_{s *}$, with $c_{s *}=\sqrt{T_{e *} / m_{i}}$ and the mass ratio $m_{i} / m_{e}$. Figure 11 plots plasma profiles for xenon, $u_{i 0} / c_{s *}=1$, and different $z_{0}$. Observe, first, that $T_{e 0} \simeq T_{e *}$ indicating that $f_{e}\left(z_{0}\right)$ is fully Maxwellian except for the high-energy tail of free electrons. Second, electron (collisionless) cooling is high and motivated by the emptying of regions in the electron velocity space [34]. Third, the total drop of the electric potential in the divergent nozzle for a current-free xenon plasma is about 7.4.

Figure 12 plots an "equivalent" local polytropic coefficient defined as

$$
\gamma=\frac{d \ln p_{e}}{d \ln n_{e}}
$$

- which presents large variations along the plume. The main message is then that there is not a polytropic behavior of the electrons. With this precaution taken, it still can be said that near the source electrons are 


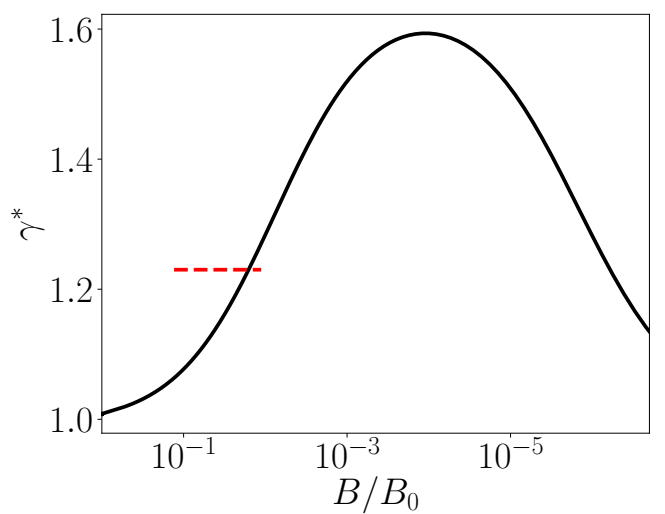

Figure 12: Equivalent local polytropic coefficient given by the model (-). The dashed horizontal red line (-- - ) represents the average experimental value measured along the MN of the PMthruster.

near-isothermal, then tend to an adiabatic behavior, and finally to a (cold) isothermal behavior again. Observe that these changes occur along a range of $B$ much larger than the experimental one.

Before experimental data is fitted to this model in the next Subsection, it is worth to highlight the main limitations of this ideal model. First, the model is paraxial and ignores partially the radial plasma expansion and totally the turning-back of magnetic lines. Second, it considers ions and electrons fully-magnetized down to $z_{\infty}$, so, in practice, results are valid only in nozzle near-region. Third, the electron temperature at the source is assumed isotropic, which is not the situation expected in an ECR thruster. Theoretical work is underway to cancel or at least reduce these model restrictions [34,36-38]

\section{IV.2 Fitting of experimental data along the MN of the PM thruster}

The experimental results for the PM thruster presented in Section III are here fitted to the model just described. For a given mass flow, the fitting method is applied to the ion velocity profile experimental data of figure 9 (a) and consists in the following. A range of values of $T_{e} *$ is selected; remember that $T_{e *} \approx T_{e 0}$. For each $T_{e *}$, the experimental point where $u_{i}=c_{s *}$ is taken as origin $z_{0}$ for the fitted curve and the full theoretical curve is plotted. Figure 13 shows the model prediction for three different values of $T_{e *}$ overlapped with the experimental data, for $2 \mathrm{sccm}$ flow. There is clearly a significant sensitivity to $T_{e *}$. A Least Square Error method is implemented to select the optimal $T_{e *}$. The ion velocity profiles were used for this fitting process because, first, LIF diagnostics allow to perform measurements from the thruster cavity to far downstream and, second, the uncertainty in the measurement of ion velocity is lower than the one regarding plasma density or electron temperature.

The optimal $T_{e}$ for each of the experimental curves $(1,1.5$ and $2 \mathrm{sccm})$ is shown with its corresponding sonic location $z_{0}$ in Table 1 (two last columns). Observe first, that the fitted sonic point is located around 1 $\mathrm{cm}$ from the thruster exit plane and shifts slightly downstream when increasing the mass flow rate. Second, the fitted electron temperature at the sonic point $T_{e 0} \approx T_{e *}$ decreases with the mass flow rate, a trend in agreement with experimental evidence [27].

Figure 14 compares the experimental and best-fitted theoretical curves for the ion velocity, the plasma potential, the electron density and the electron temperature. Figure 14 (a) shows that the agreement is rather 


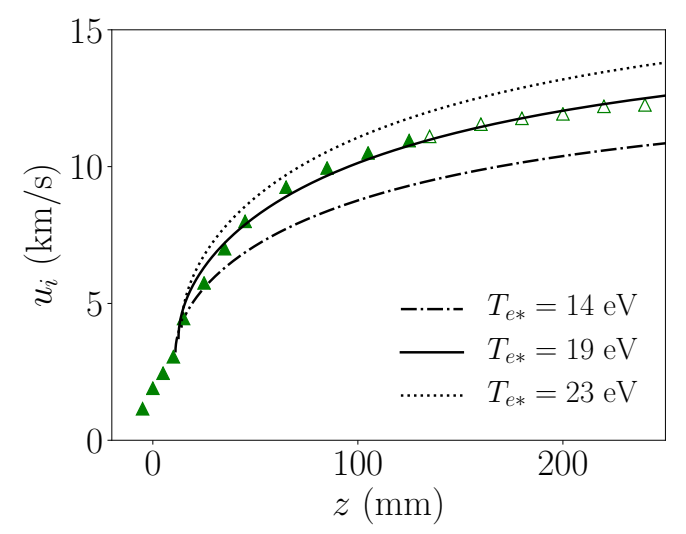

Figure 13: Experimental ion velocity curve $u_{i}(z)$ with the model predictions at different values of $T_{\text {e* }}$ for 2 sccm.

a)

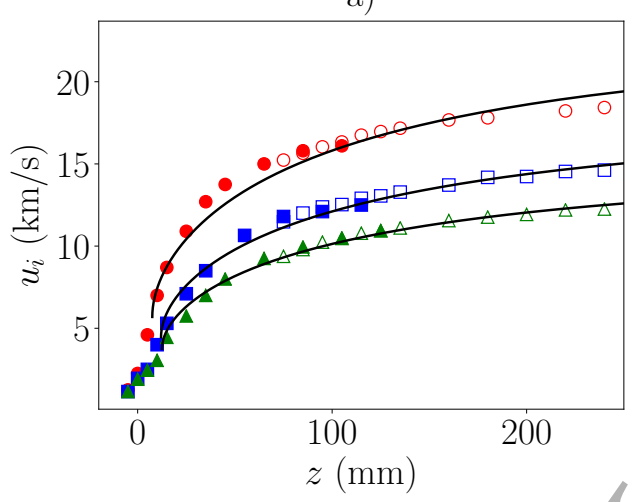

c)

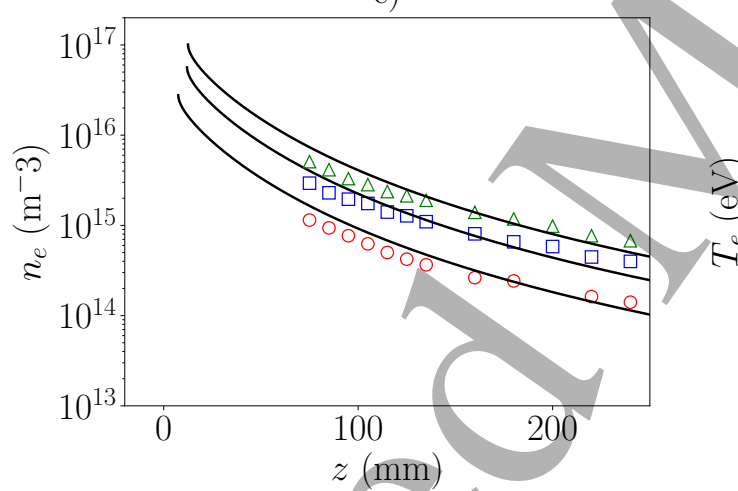

b)

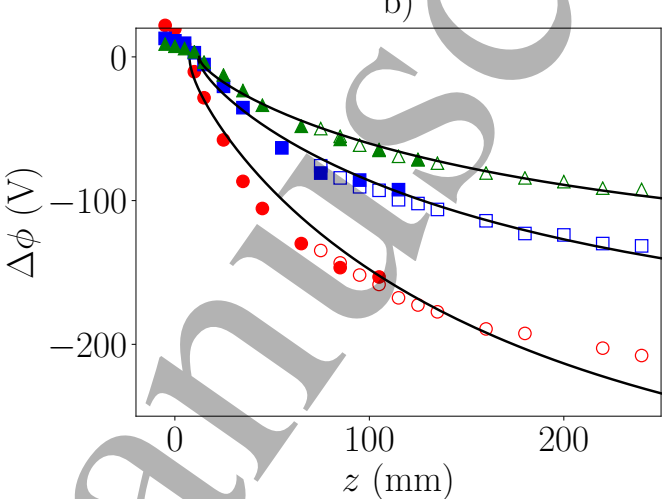
d)

50

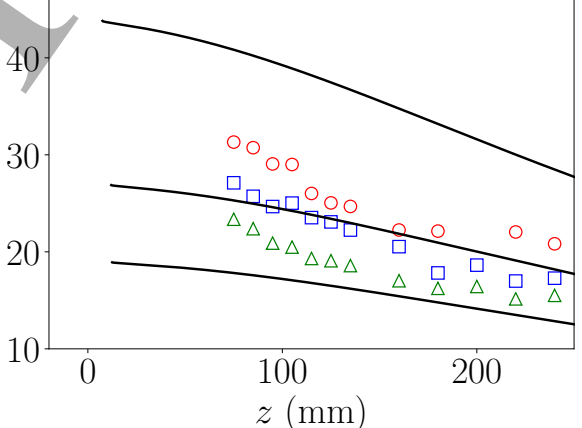

Figure 14: Plasma properties from LIF and Langmuir probe measurements compared to the quasi 1D model prediction (-) at $1 \operatorname{sccm}(\bullet, 0), 1.5 \operatorname{sccm}(\mathbf{\bullet}, \square)$ and $2 \operatorname{sccm}(\mathbf{\Delta}, \Delta)$. a) Ion velocity $u_{i}$ b) Plasma potential $\Delta \phi$ c) Electron density $n_{e}$ d) Electron temperature $T_{e}$.

good for the supersonic $u_{i}$; a bit worse for $1 \mathrm{sccm}$. Figure 14 (b) shows also a good agreement for the plasma potential. The model estimates that at the farthest measurement point $(z=250 \mathrm{~mm})$, the expansion is almost $70 \%$ complete, and therefore the ratio between the total potential drop and the estimated electron temperature is around 5, which correlates well with the values found by Lafleur et al. in [27] in the SO thruster described here.

Figure 14 (c) compares the plasma density measured with the Langmuir probe and the theoretical curves. The plasma density at the plasma reservoir $n_{0}$ has been estimated assuming flux is conserved along the mea- 
surement line (recall $n_{0} \approx n_{e *}$ ), that is $n u_{i} / B=$ const. The estimated plasma density at the sonic point $n_{0}$ is $2.77,5.56$, and 10.0 in $10^{16} \mathrm{~m}^{-3}$ units, for $1,1.5$ and $2 \mathrm{sccm}$, respectively. The model fairly describes the density drop in the measurement region, although additional data nearest the thruster exit s would be necessary to confirm the results. However, figure 14 (d) highlights that the electron temperature estimated by the model only agrees reasonably with the experimental results at $1.5 \mathrm{sccm}$, disagreeing significantly at 1 and 2 sccm. Finally, Figure 12 compares the average experimental polytropic coefficient (in a limited plume region) with the local coefficient from the model.

It can be concluded that the comparison of data and model is acceptable and promising taking into consideration the idealizations of the model. Temperature anisotropy, likely present in the plasma at the source and neglected in the MN model here, is a first major source of discrepancies here, and possibly more acute in the temperature profiles and for low mass flow rates (due to less electron-electron collisions). Work is in progress to include anisotropy in the MN model [36]. A second one is the relevance of ionization and collisional process in the real plasma plume, also neglected in the model. Incidentally, collisions would also justify that the sonic point is here placed inside the divergent nozzle and not at a MN throat [39,40]. And finally, discrepancies should not be attributed exclusively to the theory. For instance, it is acknowledged that, in the data-set at 2 sccm, the uncertainty in the Langmuir probe post-processing or the increase of the background pressure could have an impact on the results.

\section{IV.3 Differences between the PM and SO thrusters}

Measurements of the complete plasma potential profile were only performed along the MN of the PM thruster, since it was not possible to install the SO thruster in the LIF set-up. Therefore, the experimental data from the SO thruster can not be directly related to the model results. However, interesting features can be discussed. The model enhances the large variation of the local polytropic index along the expansion, a consequence of the collisionless cooling of the different electron subpopulations. The pysical mechanisms that populate the doubly-trapped electron/regions are a current subject of study; two of them are collisionality and variations of the plasma potential profile during the transient set-up [37]. The 2D magnetic field topology may influence the formation of the doubly-trapped regions, and therefore have a direct impact in the local electron cooling. As commented before, Kim et al. [24, 25] also found different polytropic indices when varying the strength and configuration of the magnetic nozzle.

\section{CONCLUSIONS}

Experimental measurements of ion acceleration and plasma properties along the MN of two ECR thruster prototypes have been performed. The difference between both versions relies on the MN topology, one generated by a permanent magnet (PM) and the other by a set of solenoids (SO). Langmuir probe measurements along the thruster's axis at several mass flow rates have added insight of the plasma response at different upstream conditions. The dependence of the total plasma potential drop with the mass flow rate has been identified, as well as a significant electron cooling rate along the expansion.

In the PM thruster set-up, simultaneously with the Langmuir probe, laser induced fluorescence (LIF) diagnostics have been performed to obtain the mean ion velocity from the thruster cavity to $125 \mathrm{~mm}$ downstream. 
By assuming total ion energy is conserved, it has been possible to estimate the complete profile of plasma potential from the thruster exit to far downstream overlapping Langmuir probe and LIF measurements. Close to the thruster cavity, a broadening of the ion distribution function has been observed, which could be due to the Zeeman splitting effect. Further experiments are however needed to fully understand this outcome.

In parallel, a quasi-1D stationary fluid-kinetic model has been adapted to a fully divergent magnetic field, assuming quasineutrality and current-free expansion. The ion population is treated as a cold fluid species, while the electron distribution function is solved self-consistently accounting for magnetic mirror effects and electric potential barriers. The unique solution for the ambipolar potential profile depends only on the ion to electron mass ratio $m_{i} / m_{e}$ and the initial ion Mach number. A total potential drop of 7.4 times the upstream electron temperature is found for xenon.

A least square error method has been implemented to compare the experimental results with the theoretical model. With this method, it has been found that the ion velocity, plasma potential and electron density profiles for different mass flow rates fit well with the model. The highest error between the model and the experimental data has been found at low mass flow rates and for the temperature profiles, which is probably related to the anisotropy of the electron energy distribution function already at the plasma reservoir plus the effects in the expansion of ionization and other collisional effects. A next step in this research will be to improve the model to incorporate electron temperature anisotropy in the plasma source and collisional effects.

On the experimental side, further work should address additional non-intrusive measurements close to the thruster exit, since for this ECR thruster prototype, the main acceleration region is concentrated in the first $10 \mathrm{~cm}$ from the thruster exit plane. Additionally, other diagnostics capable of detecting anisotropic plasma properties would definitely add insight to this research.

\section{ACKNOWLEDGMENTS}

The authors want to thank Mick Wijnen for his insightful comments and discussions. A preliminary version of this manuscript obtained the best PhD Communication Award at the Space Propulsion Conference 2018, 14-18 May, Seville. This work was made in the framework of project MINOTOR that has received funding from the European Union's Horizon 2020 research and innovation programme under grant agreement No 730028. Minor support came from the Spanish R \& D National Plan (Grant No. PN ESP2016-75887).

\section{REFERENCES}

[1] Winglee, R., Ziemba, T., Giersch, L., Prager, J., Carscadden, J., and Roberson, B., "Simulation and laboratory validation of magnetic nozzle effects for the high power helicon thruster," Physics of Plasmas, Vol. 14, No. 6, 2007, pp. 063501.

[2] Charles, C., Boswell, R., and Lieberman, M., "Xenon ion beam characterization in a helicon double layer thruster," Applied Physics Letters, Vol. 89, No. 26, 2006, pp. 261503.

[3] Takahashi, K., Charles, C., Boswell, R., and Ando, A., "Effect of magnetic and physical nozzles on plasma thruster performance," Plasma Sources Science and Technology, Vol. 23, No. 4, 2014, pp. 044004. 
[4] Pavarin, D., Ferri, F., Manente, M., Curreli, D., Guclu, Y., Melazzi, D., Rondini, D., Suman, S., Carlsson, J., Bramanti, C., Ahedo, E., Lancellotti, V., Katsonis, K., and Markelov, G., "Design of 50W Helicon Plasma Thruster," 31th International Electric Propulsion Conference, IEPC 2009-205, 2009.

[5] Batishchev, O., "Minihelicon plasma thruster," Plasma Science, IEEE Transactions on, Vol. 37, No. 8, 2009, pp. 1563-1571.

[6] Diaz, F. and Chang, R., "The VASIMR rocket," Scientific American, Vol. 283, No. 5, 2000, pp. $90-97$.

[7] Arakawa, Y. and Sasoh, A., "Steady-state permanent magnet magnetoplasmadynamic thruster," Journal of Propulsion and Power, Vol. 5, 1989, pp. 301-304.

[8] Krülle, G., Auweter-Kurtz, M., and Sasoh, A., "Technology and application aspects of applied field magnetoplasmadynamic propulsion," J. Propulsion and Power, Vol. 14, No. 5, 1998, pp. 754-763.

[9] Sercel, J., "Simple model of plasma acceleration in a magnetic nozzle," 21st International Electric Propulsion Conference, Vol. 1, 1990.

[10] Jarrige, J., Elias, P.-Q., Cannat, F., and Packan, D., "Characterization of a coaxial ECR plasma thruster," 44th AIAA Plasmadynamics and Lasers Conference, San Diego, 2013.

[11] Takahashi, K. and Ando, A., "Laboratory Observation of a Plasma-Flow-State Transition from Diverging to Stretching a Magnetic Nozzle," Physical Review Letters, Vol. 118, No. 22, 2017, pp. 225002.

[12] Arefiev, A. V. and Breizman, B. N., "Magnetohydrodynamic scenario of plasma detachment in a magnetic nozzle," Physics of Plasmas, Vol. 12, No. 4, 2005, pp. 043504.

[13] Deline, C., Bengtson, R., Breizman, B., Tushentsov, M., Jones, J., Chavers, D., Dobson, C., and Schuettpelz, B., "Plume detachment from a magnetic nozzle," Physics of Plasmas, Vol. 16, No. 3, 2009, pp. 033502.

[14] Olsen, C., Ballenger, M., Carter, M., Chang Diaz, F., Giambusso, M., Glover, T., Ilin, A., Squire, J., Longmier, B., Bering, E., and Cloutier, P., "Investigation of Plasma Detachment From a Magnetic Nozzle in the Plume of the VX-200 Magnetoplasma Thruster," Plasma Science, IEEE Transactions on, Vol. 43, No. 1, 2015, pp. 252-268.

[15] Ahedo, E. and Merino, M., "On plasma detachment in propulsive magnetic nozzles," Physics of Plasmas, Vol. 18, No. 5, 2011, pp. 053504.

[16] Ahedo, E. and Merino, M., "Two-dimensional supersonic plasma acceleration in a magnetic nozzle," Physics of Plasmas, Vol. 17, No. 7, 2010, pp. 073501.

[17] Longmier, B., Bering, E., Carter, M., Cassady, L., Chancery, W., Díaz, F., Glover, T., Hershkowitz, N., Ilin, A., McCaskill, G., et al., "Ambipolar ion acceleration in an expanding magnetic nozzle," Plasma Sources Science and Technology, Vol. 20, 2011, pp. 015007.

[18] Arefiev, A. and Breizman, B., "Ambipolar acceleration of ions in a magnetic nozzle," Physics of Plasmas, Vol. 15, No. 4, 2008, pp. 042109. 
[19] Takahashi, K., Lafleur, T., Charles, C., Alexander, P., Boswell, R., Perren, M., Laine, R., Pottinger, S., Lappas, V., Harle, T., et al., "Direct thrust measurement of a permanent magnet helicon double layer thruster," Applied Physics Letters, Vol. 98, 2011, pp. 141503.

[20] Pottinger, S., Lappas, V., Charles, C., and Boswell, R., "Performance characterization of a helicon double layer thruster using direct thrust measurements," Journal of Physics D: Applied Physics, Vol. 44, No. 23, 2011, pp. 235201.

[21] Vialis, T., Jarrige, J., Aanesland, A., and Packan, D., "Direct thrust measurement of an electron cyclotron resonance plasma thruster," Journal of Propulsion and Power, Vol. 34, No. 5, 2018, pp. 1323-1333.

[22] Takahashi, K., Charles, C., Boswell, R., and Ando, A., "Adiabatic Expansion of Electron Gas in a Magnetic Nozzle," Physical review letters, Vol. 120, No. 4, 2018, pp. 045001.

[23] Little, J. and Choueiri, E., "Electron Cooling in a Magnetically Expanding Plasma," Physical Review Letters, Vol. 117, No. 22, 2016, pp. 225003.

[24] Kim, J. Y., Chung, K., Kim, S., Ryu, J. H., Chung, K.-J., and Hwang, Y., "Thermodynamics of a magnetically expanding plasma with isothermally behaving confined electrons," New Journal of Physics, Vol. 20, No. 6, 2018, pp. 063033.

[25] Kim, J. Y., Jang, J. Y., Chung, K., Chung, K.-J., and Hwang, Y., "Time-dependent kinetic analysis of trapped electrons in a magnetically expanding plasma," Plasma-Sources Science and Technology, Vol. 28, No. 07LT01, 2019.

[26] Cannat, F., Lafleur, T., Jarrige, J., Chabert, P., Elias, P.-Q., and Packan, D., "Optimization of a coaxial electron cyclotron resonance plasma thruster with an analytical model," Physics of Plasmas, Vol. 22, No. 5, 2015, pp. 053503.

[27] Lafleur, T., Cannat, F., Jarrige, J., Elias, P., and Packan, D., "Electron dynamics and ion acceleration in expanding-plasma thrusters," Plasma Sources Science and Technology, Vol. 24, No. 6, 2015, pp. 065013.

[28] Jarrige, J., Correyero Plaza, S., Elias, P.-Q., and Packan, D., "Investigation on the ion velocity distribution in the magnetic nozzle of an ECR plasma thruster using LIF measurements," 2017.

[29] Martínez-Sánchez, M., Navarro-Cavallé, J., and Ahedo, E., "Electron cooling and finite potential drop in a magnetized plasma expansion," Physics of Plasmas, Vol. 22, No. 5, 2015, pp. 053501.

[30] Hutchinson, I. H., "Principles of plasma diagnostics," Plasma Physics and Controlled Fusion, Vol. 44, No. 12, 2002, pp. 2603.

[31] Druyvesteyn, M. and Penning, F. M., "The mechanism of electrical discharges in gases of low pressure," Reviews of Modern Physics, Vol. 12, No. 2, 1940, pp. 87.

[32] Zhang, Y., Charles, C., and Boswell, R., "Thermodynamic study on plasma expansion along a divergent magnetic field," Physical review letters, Vol. 116, No. 2, 2016, pp. 025001. 
[33] Brown, D. L., Walker, M. L., Szabo, J., Huang, W., and Foster, J. E., "Recommended practice for use of Faraday probes in electric propulsion testing," Journal of Propulsion and Power, Vol. 33, No. 3, 2016, pp. $582-613$.

[34] Merino, M., Mauriño, J., and Ahedo, E., "Kinetic electron model for plasma thruster plumes," Plasma Sources Science and Technology, Vol. 27, No. 3, 2018, pp. 035013.

[35] Navarro-Cavallé, J., Correyero, S., and Ahedo, E., "Collisionless electron cooling on magnetized plasma expansions: advances on modelling," $34^{\text {th }}$ International Electric Propulsion Conference, paper 2015-117, Electric Rocket Propulsion Society, Fairview Park, OH, Kobe, Japan, July 6-10, 2015.

[36] Correyero, S., Navarro-Cavallé, J., and Ahedo, E., "Expansion of a collisionless magnetized plasma plume with bi-Maxwellian electrons," 52nd Joint Propulsion Conference, AIAA, Reston, VA, Salt Lake City, Utah, July 25-27, 2016, p. 5035.

[37] Sánchez-Arriaga, G., Zhou, J., Ahedo, E., Martínez-Sánchez, M., and Ramos, J. J., "Kinetic features and non-stationary electron trapping in paraxial magnetic nozzles," Plasma Sources Science and Technology, Vol. 27, No. 3, 2018, pp. 035002.

[38] Ramos, J., Merino, M., and Ahedo, E., "Three dimensional fluid-kinetic model of a magnetically guided plasma jet," Physics of Plasmas, Vol. 25, No. 6, 2018, pp. 061206.

[39] Burm, K., Goedheer, W., and Schram, D., "Mach numbers for gases and plasmas in a convergent-divergent cascaded arc," Physics of Plasmas, Vol. 6, No. 6, 1999, pp. 2628-2635.

[40] Manheimer, W. and Fernsler, R., "Plasma Acceleration by Area Expansion," IEEE Transactions on Plasma Science, Vol. 29, 2001, pp. 75-84. 